\title{
PRODUÇÃO DE BIOMASSA DA CANA-DE-AÇÚCAR POR MEIO DO USO DE ORGANOMINERAIS EM CANA PLANTA E CANA SOCA
}

\author{
Camila Andrea de Souza ${ }^{1 *}$, André César Vitti ${ }^{2}$, Carlos Roberto Sanquetta ${ }^{3}$, Edilaine Cova \\ Gaitarossa ${ }^{4}$ \\ 1* Universidade Federal do Paraná, Pós-graduação em Bioenergia, Curitiba, Paraná, Brasil, \\ camilaandreasouza@gmail.com \\ ${ }^{2}$ Agência Paulista de Tecnologia dos Agronegócios, Piracicaba, São Paulo, Brasil, acvitti@apta.sp.gov.br \\ ${ }^{3}$ Universidade Federal do Paraná, Departamento de Ciências Florestais, Curitiba, Paraná, Brasil, \\ carlos sanquetta@hotmail.com \\ ${ }^{4}$ SynTech Research, Piracicaba, São Paulo, Brasil, gaitarossa@syntechresearch.com
}

\begin{abstract}
Resumo
A área cultivada com cana-de-açúcar (Saccharum spp.) vem aumentando nos últimos anos, o que torna necessário realizar estudos para verticalizar a sua produção, em que o emprego de novas tecnologias pode proporcionar ganhos de produtividade nos canaviais. Nesse sentido, o presente trabalho objetivou avaliar o desenvolvimento da cana-de-açúcar, tanto em cana planta como em cana soca (cultivar SP81-3250), com o uso dos produtos organominerais, associados ou não, comparados com o uso da torta de filtro. O experimento foi desenvolvido em vaso (cerca de $50 \mathrm{~kg}$ de solo de textura arenosa), em Piracicaba/SP, durante oito meses, cerca de 120 dias por ciclo. O delineamento foi em blocos casualizados, com nove tratamentos e três repetições em cana planta, enquanto em cana soca foi utilizado esquema fatorial $2 \times 5$, com cinco tratamentos em dois níveis (com e sem aplicação de organomineral) e três repetições. De acordo com os resultados obtidos, pode-se averiguar que, não houve diferença significativa entre os tratamentos com organomineral e a torta de filtro no acúmulo de fitomassa na parte aérea e no sistema radicular em cana planta, ao passo que a aplicação de organomineral mostrou-se vantajosa para o aumento de matéria seca do sistema radicular em cana soca.
\end{abstract}

Palavras-chave: Ácidos orgânicos; Fitomassa; Produtividade.

\begin{abstract}
Biomass production of sugarcane by organo-mineral use in plant cane and soca cane. The area cultivated with sugarcane (Saccharum spp.) has been increasing in recent years, making it necessary to conduct studies to verticalize its production, where the use of new technologies can provide productivity gains in the cane fields. For this, the present study aimed to evaluate the development of sugarcane in both plant cane and soca cane (cultivar SP81-3250), with the use of organo-mineral, associated or not, compared to the use of pie filter. The experiment was conducted in pots (about $50 \mathrm{~kg}$ of sandy soil texture) in Piracicaba/SP, for eight months, about 120 days per cycle. The design was a randomized block design with nine treatments and three replicates in plant cane, while in soca cane was used factorial $2 \times 5$, with five treatments at two levels (with and without application of organo-mineral) and three replications. According to the results, there was non-significant difference between treatments with organo-mineral and the filter cake in the dry matter accumulation in above ground and root systems in plant cane, while the application of organo-mineral showed advantageous to increase the dry matter of root system in soca cane.
\end{abstract}

Keywords: Organic acids; Phytomass; Productivity.

\section{INTRODUÇÃO}

Nas últimas safras, a produtividade de cana-de-açúcar apresentou queda em razão de eventos climáticos (seca prolongada na safra 2010/2011, seca e geada na safra 2011/2012); baixa qualidade de mudas; adubação abaixo dos níveis ideais; aumento de pragas e doenças; baixa taxa de renovação nos canaviais, aumentando a média de idade das plantas no campo; 
fase de adaptação de áreas de plantio convencional com incentivo à colheita mecanizada; falta de conhecimento mais detalhado, principalmente nas novas fronteiras agrícolas, envolvendo manejos nutricional, varietal, épocas de plantio e colheita. Em relação aos ambientes de produção, verifica-se que regiões tradicionalmente ocupadas com pastagens e de baixa fertilidade estão sendo utilizadas para cultivo de cana-de-açúcar. Para remediar os efeitos negativos, é necessário realizar o manejo associado com a novas tecnologias (VITTI et al., 2009).

Segundo Doran (1997) e Reeves (1997), a matéria orgânica (MO) é o principal indicador da qualidade do solo, pois seu teor é muito sensível às práticas de manejo. Segundo Beauclair et al. (2007), a composição da MO pode ser simulada pelos chamados organominerais, fontes de ácidos húmicos e fúlvicos que, segundo Vitti et al. (2009), podem estimular o desenvolvimento do sistema radicular da cultura, interferindo diretamente no aspecto nutricional e no vigor da planta.

Desta forma, por meio do presente trabalho, objetivou-se estudar em vaso de $50 \mathrm{~kg}$ de terra os efeitos da utilização de organominerais, tanto em cana planta como no ciclo seguinte de cana soca, quanto ao parâmetro matéria seca da parte aérea e do sistema radicular.

\section{MATERIAL E MÉTODOS}

O experimento foi desenvolvido em Piracicaba/SP, em local com coordenadas geográficas $22^{\circ} 42^{\prime} 30^{\prime \prime}$ Sul e $47^{\circ} 38^{\prime} 00^{\prime \prime}$ Oeste e altitude de $546 \mathrm{~m}$. O estudo foi realizado em vasos ao ar livre, contendo $50 \mathrm{~kg}$ de um substrato com origem da camada superficial de um Argissolo, coletado à profundidade de 0-0,4 m em área de reforma de canavial de textura arenosa (90\% de areia). A variedade de cana-de-açúcar foi a SP81-3250, com o plantio de mudas transplantadas em julho de 2010, a fim de obter maior uniformidade de plantas, cujas colheitas foram realizadas em duas épocas: final de novembro de 2010, em cana planta (parte aérea e/ou sistema radicular); e início de abril de 2011, em cana soca (parte aérea e sistema radicular).

$\mathrm{O}$ solo coletado apresentou as seguintes características químicas (0-0,2 m e 0,2-0,4 m): $\mathrm{pH} 5,8 \backslash 5,3 \mathrm{em} \mathrm{CaCl} 2 ; 18 \backslash 13 \mathrm{mg} \mathrm{dm}{ }^{-3}$ de P (resina); K, Ca, Mg, H+Al, Al, CTC: 1,3\1,3, 13\13, 6\5,

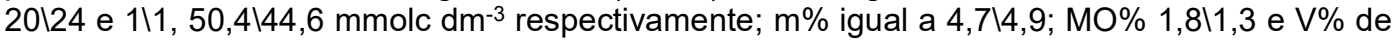
$50,4 \backslash 44,6$. Na ocasião do plantio, o solo foi peneirado e corrigido a uma saturação por bases (V\%) de 80. Os corretivos foram aplicados separadamente em cada vaso (calcário dolomítico na dose de $2 \mathrm{t} \mathrm{ha}^{-1} \mathrm{de} \mathrm{p} / \mathrm{v}$ homogeneizando com o solo. A torta de filtro do experimento foi composta de $64 \%$ de $\mathrm{MO}, 33 \%$ de carbono orgânico, $52 \%$ de umidade, $1,3 \%$ de $\mathrm{N}$-total, $1,6 \%$ de $\mathrm{P}_{2} \mathrm{O}_{5} \mathrm{e}$ $0,3 \%$ de $\mathrm{K}_{2} \mathrm{O}$.

Os produtos utilizados no experimento foram o organomineral $\mathrm{H}(12 \%$ de ácidos húmicos; $3 \%$ de fúlvicos; $5 \%$ de $\mathrm{K}_{2} \mathrm{O}$ e $8 \%$ de $\mathrm{N}$; aspecto líquido de cor preta com $\mathrm{pH} 13$ e densidade $\left.1,1 \mathrm{~g} / \mathrm{cm}^{3}\right)$, associado ou não ao organomineral $\mathrm{R}$ (7\% de aminoácidos livres; 5,5\% de N-total; $1,5 \%$ de nitrogênio orgânico; $5 \%$ de $\mathrm{P}_{2} \mathrm{O}_{5} ; 3,5 \%$ de $\mathrm{K}_{2} \mathrm{O} ; 15 \%$ de $\mathrm{MO} ; 0,036 \%$ de $\mathrm{Fe}-$ EDDHA; 0,05\% de Mn-EDTA; 0,07\% de Zn-EDTA; 0,10\% de Mo; aspecto líquido de coloração vermelha com $\mathrm{pH} 4,2 \mathrm{e}$ densidade $1,2 \mathrm{~g} \mathrm{~cm}^{-3}$ ). Embora os organominerais apresentassem macronutrientes em sua composição, não houve adição complementar nos tratamentos que não receberam os produtos, devido a pequena quantidade adicionada.

A adubação com macro e micronutrientes foi igual para todos os vasos do experimento e calculada em função do volume de solo por vaso, sendo adicionada no plantio de acordo com a quantidade recomendada pelo Boletim 100 (ESPIRONELLO et al., 1996). Exceto para N, P e $\mathrm{K}$, cujas quantidades foram calculadas de acordo com material seco acumulado esperado em cada vaso (equivalente a $1 \mathrm{~kg}$ de massa de material seco por vaso), sendo em torno de 56 e $50 \mathrm{~g}$, respectivamente, na forma de ureia $(45 \%$ de $\mathrm{N})$ e cloreto de potássio $\left(63 \%\right.$ de $\left.\mathrm{K}_{2} \mathrm{O}\right)$ e $50 \mathrm{~g}$ de supersimples $\left(18 \%\right.$ de $\left.\mathrm{P}_{2} \mathrm{O}_{5}\right)$.

Os micronutrientes adicionados para evitar possíveis deficiências dos elementos, e consequentemente limitação do desenvolvimento da cultura, foram: $8 \mathrm{~kg} \mathrm{ha}^{-1} \mathrm{de} \mathrm{Zn}$; $1 / 2 \mathrm{~kg} \mathrm{ha}^{-1} \mathrm{de}$ $\mathrm{Cu} ; 2 \mathrm{~kg} \mathrm{ha}{ }^{-1}$ de $\mathrm{B}$ e $1 / 2 \mathrm{~kg} \mathrm{ha}^{-1}$ de $\mathrm{Mn}$. Os micros e macronutrientes, aplicados na forma líquida e sólida, respectivamente, foram colocados cerca de $2 \mathrm{~cm}$ abaixo do local em que as mudas ficariam e uma fina camada de solo cobriu tais nutrientes.

Os produtos $\mathrm{H}$ e $\mathrm{R}$, foram aplicados sobre o tolete no momento do plantio em cana planta e logo após o corte em cana soca. Em cana planta também foram aplicados produtos que controlam pragas de solo Furadan (Carbofuran) e Regent (Fipronil), com intuito de não prejudicar o desenvolvimento do sistema radicular, não interferindo no efeito dos tratamentos.

Os produtos organominerais, assim como os micronutrientes, foram diluídos separadamente em água deionizada nos balões volumétricos e aplicados por meio de proveta 
graduada, para melhor quantificação e distribuição das doses estabelecidas para cada vaso. Em cana soca houve uma adubação após 35 dias do corte da cana planta, com aplicação em cobertura na dose de $250 \mathrm{~g}$ do formulado 20-05-20 (N-P-K). Cerca de quatro (cana planta) e nove meses (cana soca) após o transplantio das mudas de cana-de-açúcar, foram realizadas as colheitas.

Em cana planta, o delineamento experimental foi em blocos casualizados, com nove tratamentos e três repetições: T1-Testemunha (Test): sem adição dos produtos organominerais; T2-10H: Adição de $10 \mathrm{~L}_{\text {ha-1 }}$ de organomineral H; T3-10 H+1R: $10 \mathrm{~L}^{-1}$ de organomineral H + $1 \mathrm{~L} \mathrm{ha}^{-1}$ de organomineral R; T4-10 H+2R: $10 \mathrm{~L} \mathrm{ha}^{-1}$ de organomineral $\mathrm{H}+2 \mathrm{~L} \mathrm{ha}^{-1}$ de organomineral R; T5-20 H: $20 \mathrm{~L}^{-1}$ de organomineral $\mathrm{H}$; T6-20 H+1R: $20 \mathrm{~L}^{-1} \mathrm{ha}^{-1}$ de organomineral $\mathrm{H}+1 \mathrm{~L} \mathrm{ha}^{-1}$ de organomineral $\mathrm{R}$; T7-20 H+2R: $20 \mathrm{~L} \mathrm{ha}^{-1}$ de organomineral $\mathrm{H}+2$ $\mathrm{L}$ ha-1 de organomineral R; T8-2R: $2 \mathrm{~L} \mathrm{ha}^{-1}$ de organomineral R; T9-torta de filtro $10 \mathrm{t} \mathrm{ha}^{-1} \mathrm{em}$ base seca (tratamento referência).

Em cana planta, coletou-se a parte aérea (PA) de todos os tratamentos e o sistema radicular de T2, T4 e T6. Esses tratamentos, por terem apenas três vasos cada um, não apareceram na cana soca. Já os tratamentos T5, T7 e T8 que foram realizados em duplicata (total de seis vasos cada tratamento) e não tiveram o sistema radicular retirados, foram mantidos para estudar na soca o efeito da aplicação ou não aplicação de $20 \mathrm{~L} \mathrm{ha}^{-1}$ de organomineral $\mathrm{H}$ mais $2 \mathrm{~L} \mathrm{ha}^{-1}$ de organomineral R. No T1 e T9, houve plantio em triplicata (total de nove vasos cada tratamento). Assim, pode-se coletar o sistema radicular em cana planta e estudar na soca a aplicação ou não aplicação dos produtos organominerais como visto acima. No T3, não foi colhido sistema radicular, mas não foi mantido para a soca.

Em cana soca, foi realizado estudo da PA e SR dos tratamentos remanescentes da cana planta T1, T5, T7, T8 e T9 no delineamento experimental em blocos casualizados em esquema fatorial $2 \times 5$, com dois níveis (com e sem aplicação de $20 \mathrm{~L} \mathrm{ha}^{-1}$ de organomineral $(\mathrm{H})$ mais $2 \mathrm{~L}$ ha $^{-1}$ de organomineral $\left.(R)\right)$ e 5 tratamentos (T1, T5, T7, T8 e T9) em 3 repetições.

A parte aérea de cada vaso, inicialmente, foi pesada (peso úmido) e em seguida triturada. Após a trituração foi sub-amostrada, colocada em estufa a $60^{\circ} \mathrm{C}$ por 72 horas para a determinação da umidade. O sistema radicular (raízes e rizomas) de cada vaso foi lavado em peneiras, para retirada total do solo e, em seguida, separados e colocados em estufa. Após a secagem da parte aérea e do sistema radicular, quantificou-se a massa de material seco produzido em cada vaso.

Os valores obtidos das variáveis de respostas foram submetidos a análise de variância, utilizando o teste $\mathrm{F}$ ao nível de $95 \%$ de confiança. Posteriormente, para as causas de variação significativas, foi aplicado o teste de significância de Tukey $(p=0,05)$ utilizando o programa SAS.

\section{RESULTADOS E DISCUSSÃO}

\section{Cana planta}

Ao avaliar o acúmulo de massa de material seco na parte aérea, foi verificado que nos tratamentos que receberam produtos organominerais ou torta de filtro, os valores foram superiores cerca de três vezes ao tratamento testemunha (Figura 1). Foi possível averiguar que não houve diferença entre os tratamentos com os organominerais em relação ao que recebeu a torta de filtro. Assim, o produto organomineral como a torta de filtro (T9) foi eficiente em produzir fitomassa na parte aérea (PA).

Em relação ao acúmulo de fitomassa no sistema radicular (SR), foi verificado diferença entre os tratamentos estudados em relação à testemunha, que foi, em média, cerca de 2,4 vezes superior (Figura 2). Praticamente não houve diferença dos tratamentos com os produtos organominerais em relação a torta de filtro. O sistema radicular apresenta menor número de tratamentos devido ter sido avaliado apenas do T1, T2, T4, T6 e T9. O fato de o estudo ter sido em vaso, o espaço restrito poderia ter limitado o desenvolvimento das raízes, não sendo possível verificar diferença entre tratamentos com os organominerais, mas o maior desenvolvimento das raízes com esses tratamentos em relação à testemunha demonstra que isso não ocorreu. Por essas observações, o uso de produtos organominerais podem contribuir para um sistema radicular mais desenvolvido e, caso confirmado ao nível de campo, poderá possibilitar um canavial com alto potencial produtivo e, consequentemente, obter maior longevidade das socas. 


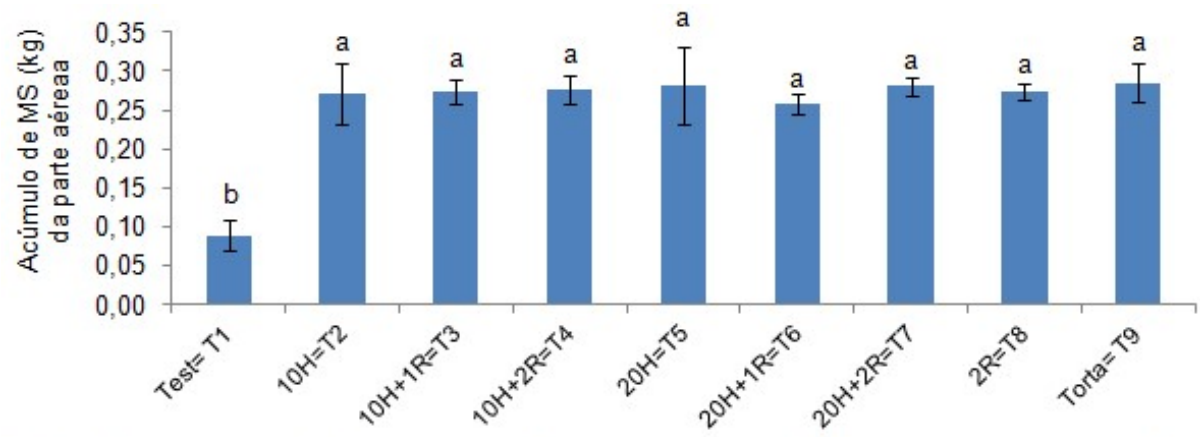

Test= Testemunha ; $\mathrm{H}=\mathrm{Litros}$ de organomineral $\mathrm{H}$; $\mathrm{R}=\mathrm{Litros}$ de organomineral $\mathrm{R}$; Médias seguidas da mesma letra não diferem pelo teste de tukey; ${ }^{*}$ significativo ao nível de $1 \%$ de probabilidade $(p<.01) ; C . V(\%) 11,13 \%$; Valor $F$ 15,06*; $\mathrm{PROB} .>\mathrm{F}<0,0001$

Figura 1. Acúmulo de matéria seca da parte aérea em cana planta.

De acordo com Vitti et al. (2009), em experimento realizado em vaso estudando cana planta, demonstraram que não houve diferença significativa para acúmulo de fitomassa na planta toda entre tratamentos que receberam produtos organominerais (três tratamentos com doses de 1,5; 3,0 e 4,5 $\mathrm{L} \mathrm{ha}^{-1}$ ) comparado ao tratamento com torta de filtro (dose de $20 \mathrm{t} \mathrm{ha}^{-1}$ ). Verificaram também o acúmulo de nutrientes na cana planta e concluíram que os organominerais conseguiram ser mais eficientes em produzir fitomassa comparados à torta em relação a quantidade de nutriente absorvida. Franco et al. (2007), citados por Vitti et al. (2009), comenta uma possível explicação baseada no consumo de luxo, pois, apesar de ocorrer maior acúmulo de nutrientes na torta de filtro, tanto na parte aérea como no sistema radicular, o nutriente absorvido pode não ter sido totalmente metabolizado, havendo gasto desnecessáro de energia/nutrientes.

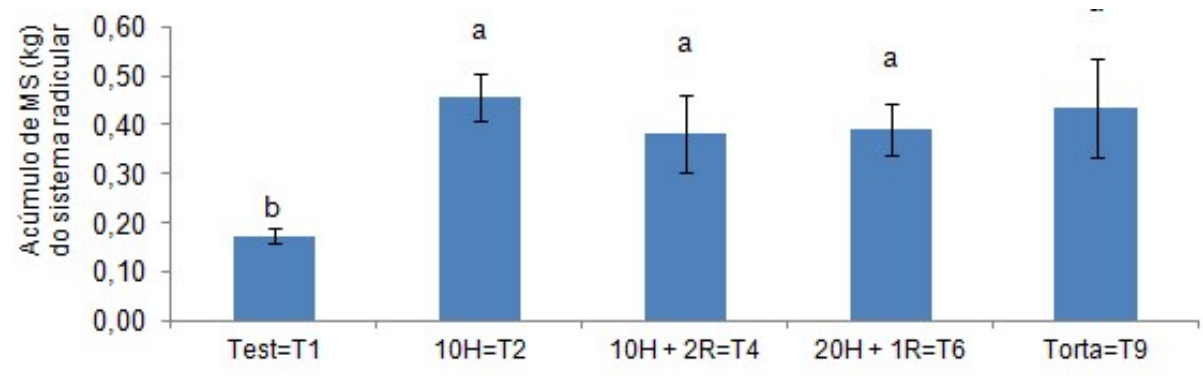

Test= Testemunha ; $\mathrm{H}=$ Litros de organomineral $\mathrm{H} ; \mathrm{R}=$ Litros de organomineral $\mathrm{R}$; Médias seguidas da mesma letra não diferem pelo teste de tukey; ${ }^{*}$ significativo ao nível de $1 \%$ de probabilidade $(p<.01) ; C . V(\%) 13,62 \%$; Valor F 15,32**; PROB. $>$ F $<0,0008$.

Figura 2. Acúmulo de matéria seca do sistema radicular em cana planta.

Outros autores demonstraram benefícios na aplicação de organominerais em relação à produtividade, a qual tem relação direta com fitomassa produzida. Isso foi observado por Gullo (2006), cuja dose de 350 L. ha-1 aplicada no sulco de plantio da cana planta (variedade PO88-62) foi equivalente à aplicação de $1.200 \mathrm{~kg}$. ha-1 de adubo mineral.

\section{Cana soca}

O uso ou não de organomineral não afetou a matéria seca da parte aérea (Tabela 1), ao passo que a matéria seca de raíz apresentou valores maiores quando se teve aplicação dos organominerais na soca, demonstrando que a dose de $20 \mathrm{~L} \mathrm{ha}^{-1}$ de organomineral $(\mathrm{H})$ mais $2 \mathrm{~L}$ ha $^{-1}$ de organomineral $(R)$ foi importante para a soca ao proporcionar maior fitomassa radicular. Outros experimentos demonstraram benefícios para o sistema radicular na aplicação de organominerais, que possuem em sua composição ácidos húmicos e fúlvicos, indo de encontro com o aumento de fitomassa demonstrado na soca. 
Tabela 1. Acúmulo de matéria seca (MS) da parte aérea e do sistema radicular em cana soca

\begin{tabular}{ccc}
\hline \multirow{2}{*}{ Tratamentos } & \multicolumn{2}{c}{ Matéria Seca (MS) } \\
\cline { 2 - 3 } & Parte Aérea & Sistema Radicular \\
Aplicado & $0,84 \mathrm{a}$ & $1,16 \mathrm{a}$ \\
Não aplicado & $0,79 \mathrm{a}$ & $0,94 \mathrm{~b}$ \\
C.V (\%) & $11,08 \%$ & $25,68 \%$ \\
Valor F Organomineral & $1,76^{\mathrm{ns}}$ & $4,95^{*}$ \\
PROB.>F Organomineral & 0,19 & 0,03 \\
Valor F Tratamentox Organomineral & $1,70^{\mathrm{ns}}$ & $0,34^{\mathrm{ns}}$ \\
PROB.>F Tratamento x Organomineral & 0,19 & 0,84
\end{tabular}

Em que: A- Recebeu aplicação (A) de $20 \mathrm{~L} \mathrm{ha}^{-1} \mathrm{de} \mathrm{H} \mathrm{+} \mathrm{2L} \mathrm{ha-1}$ de R; N- Não (N) recebeu aplicação; Médias seguidas por letra minúscula diferente na vertical, dentro de cada coluna, diferem entre si pelo teste de Tukey ao nível de $5 \%$ de probabilidade $(0.01=<p<0.05)$ para a "SR".

Para Canellas et al. (2006), os ácidos húmicos tem ação sobre a formação de sítios de mitose nas raízes, aumentando raízes laterais emergidas, assim como aumento na atividade da bomba de $\mathrm{H+}$, favorecendo a emissão de pêlos radiculares, que em associação com moléculas bioativas de grupos auxínicos presentes nas substâncias húmicas, interagindo com a membrana plasmática da raiz, favoreceriam a maior absorção de água e nutrientes, o que possui ligação direta com a nutrição e produtividade da cultura.

Façanha et al. (2002) verificaram também, isolados de lodo de uma estação de tratamento de esgoto e de vermicomposto, a presença de grupamentos auxina na estrutura dos ácidos húmicos o que pode indicar a bioatividade desses ácidos e, portanto, o desenvolvimento de raízes averiguado em plântulas de café e de milho. Aguiar et al. (2009) averiguaram que comprimento de raízes laterais e eixo principal e a área radicular de plântulas de Arabidopsis e milho não estavam significativamente relacionados com a distribuição de massa molecular de ácido húmico o que pode indicar que o desenvolvimento radicular esteja mais ligado às carcterísticas bioestimulantes das substâncias húmicas.

\section{CONCLUSÕES}

De maneira geral, em cana planta, os tratamentos contendo os organominerais $(\mathrm{H}$ e/ou $\mathrm{R})$ praticamente não diferiram do tratamento contendo torta de filtro (tratamento referência) nos atributos: acúmulo de MS na parte aérea e sistema radicular. Assim, esses produtos indicam uma melhoria do vigor no desenvolvimento da cana planta na fase inicial.

$\mathrm{Na}$ cana soca em vaso, os resultados foram menos consistentes e, portanto, a utilização de organomineral parece ser mais vantajosa na fase inicial da cultura, ao passo que o aumento no parâmetro matéria seca de raíz é uma característica desejável.

\section{REFERÊNCIAS}

AGUIAR, N. O.; CANELLAS, L. P.; DOBBSS, L. B.; ZANDONADI, D. B.; OLIVARES, F. L. Distribuição de massa molecular de ácidos húmicos e promoção do crescimento radicular. Revista Brasileira de Ciência do Solo, v. 33, p. 1613-1623, 2009.

BEAUCLAIR, E. G. F.; OTAVIANO, J. A.; MALFATO, C. A. Condicionador orgânico de solo no incremento da produtividade da cana-de-açúcar. Ribeirão Preto: Idea News, 2007.

CANELLAS, L. P.; ZANDONADI, D. B.; OLIVARES, F. L.; FAÇANHA, A. R. Efeitos fisiológicos de substâncias húmicas - o estímulo às H+-ATPases. In: Nutrição Mineral de Plantas. Viçosa: Sociedade Brasileira de Ciência do Solo, 2006.

DORAN, J. W. Soil quality and sustainability. In: CONGRESSO BRASILEIRO DE CIÊNCIA DO SOLO, 26., 1997, Rio de Janeiro. Anais... Rio de Janeiro: SBCS, 1997.

ESPIRONELLO, A.; RAIJ, B.; PENATTI, C. P.; CANTARELLA, H.; MORELLI, J. L.; ORLANDO FILHO, J.; LANDELL, M. G. A.; ROSSETTO, R. Cana-de-açúcar. In: RAIJ, B.; CANTARELLA, 
H.; QUAGGIO, J. A.; FURLANI, A. M. C. Recomendações de adubação e calagem para o Estado de São Paulo. Campinas, Fundação IAC, p. 237-239, 1996.

FAÇANHA, A. R.; OKOROKOVA-FAÇANHA, A. L.; OLIVARES, F. L.; GURIDI, F.; SANTOS, G. A.; VELLOSO, A. C.x.; RUMJANEK, V. M.; BRASIL, F.; SCHRIPSEMA, J.; BRAZ-FILHO, R.; OLIVEIRA, M. A. de; CANELLAS, L. P. Bioatividade de ácidos húmicos: efeitos sobre o desenvolvimento radicular e a bomba de prótons da membrana plasmática. Pesquisa Agropecuária Brasileira, v. 37, n. 9, p. 1301-1310, 2002.

GULLO, M. J. M. Uso de condicionador de solo a base de ácido húmico na cultura da canade-açúcar (Saccaharum spp.). Dissertação (Mestrado em Fitotecnia) - Universidade de São Paulo, Coordenação de Aperfeiçoamento de Pessoal de Nível Superior, 2006.

REEVES, D. W. The role of soil organic matter in maintaining soil quality in continuous cropping systems. Soil \&Tillage Research, v. 43, p. 131-167, 1997.

VITTI, A. C.; ROSSETTO, R.; CANTARELLA, H.; NAJIM, H. M.; DIAS, F. L. F.; TEIXEIRA, R. R. Eficiência agronômica da adubação da cana planta com fonte complementar organomineral. In: II Simpósio Científico dos Pós-graduandos do CENA-USP, Piracicaba, 2009. 